\title{
El sentido liberador del juego a través del ocio*
}

\author{
Gustavo Adolfo Maldonado Martínez" \\ Jesús Erbey Mendoza Negrete"*
}

Recibido: 12 de diciembre de 2019 • Aceptado: 7 de marzo de 2020

\section{Resumen}

El presente trabajo plantea el sentido liberador del juego a través del ocio y la recreación. Partimos de los conceptos de ocio y tiempo libre como pautas para el juego y la recreación. Se realiza un análisis de la relación entre dichos conceptos, como un ejercicio que despliega el libre juego de las facultades del individuo para que su recreación no solo tenga un propósito de reposición de fuerzas, sino de recreación de sí mismo y descanso en sí mismo, con el fin de destacar sus conceptos como actividades liberadoras ante el imperativo del trabajo. Tal reflexión pone en discusión que el ocio y el juego

Artículo de reflexión. Derivado del proceso de elaboración de la tesis: El valor del ocio en la experiencia edificante, desarrollada por el maestro en Humanidades Gustavo Adolfo Maldonado Martínez, y bajo la dirección del Dr. Jesús Erbey Mendoza Negrete. En cumplimiento de los objetivos del intercambio académico en estancia de investigación en el Instituto de Estudios de Ocio de la Universidad de Deusto. Con financiación del Consejo Nacional de Ciencia y Tecnología, como parte del programa de Doctorado en Educación Artes y Humanidades de la Facultad de Filosofía y Letras de la Universidad Autónoma de Chihuahua, México.

Citar como: Maldonado, G. y Mendoza, J. (2020). El sentido liberador del juego a través del ocio. Revista de Investigación Cuerpo, Cultura y Movimiento, 10(1), 155-172. DOI: https:// doi.org/10.15332/2422474x/5966

"* Maestro en Humanidades y estudiante del cuarto semestre del Doctorado en Educación Artes y Humanidades de la Facultad de Filosofía y Letras de la Universidad Autónoma de Chihuahua, México. Correo electrónico: gustavo.maldonado4724@gmail.com; p227606@uach.mx. ORCID: https://orcid.org/0000-0001-7345-5514

"** Doctor en Filosofía de la Cultura. Docente e investigador (nivel candidato del Sistema Nacional de Investigadores) de la Facultad de Filosofía y Letras de la Universidad Autónoma de Chihuahua, México. Miembro del grupo académico de investigación en humanidades del Doctorado en Educación Artes y Humanidades de la Universidad Autónoma de Chihuhua, México. jmendozan@uach.mx. oRcID: https://orcid.org/0000-0003-2122-4516 
han de complementar el trabajo de manera armoniosa y equilibrada pero no necesariamente en función del trabajo mismo. Finalmente, se propone la comprensión del vínculo entre ocio y juego, posible a través de la recreación, no como un mero medio para descansar del trabajo, sino para el beneficio y realización de los seres humanos en sentido liberador.

Palabras clave: juego, liberación, ocio, recreación, tiempo libre. 


\section{The liberating sense of the game through leisure}

\section{Abstract}

This paper establishes the liberating sense of the game through leisure and recreation, as an exercise that unfolds the free play of an individual's capabilities so that leisure is not only a means to recover strength and energy, but also a recreation in itself and rest in itself. The starting point is an analysis of the relationship between the concepts of leisure and free time as models for the game and recreation, at work, related to the study of leisure. We set up a dialogue with other perspectives which highlight the importance of the game in cultural, educational, and aesthetic dimensions, so as to foreground their concepts as liberating activities before the imperative of work. All this provided that leisure and game are to complement work in a harmonious and balanced way, and not necessarily depending on work itself; in other words, not as a means to rest from work, but as a beneficial exercise for the realization of the human beings in a liberating sense, feasible in free time through game and leisure.

Keywords: game, liberation, leisure, recreation, free time. 
Quiero que la vida sea un juego, pero un juego en el que haya mucho en juego.

B. BLACK, La abolición del trabajo

\section{Introducción}

Distinción entre ocio y tiempo libre, pautas para la orientación del sentido del juego

Para comenzar hemos de poner a dialogar los conceptos clave que se desarrollarán, por lo que distinguiremos primeramente el ocio y el juego, que, orientados a la realización, son condición de posibilidad en un sentido liberador y recreativo para el ser humano. Por definición, el ocio tiene que ver con la liberación de la ocupación, pero también con el reposo, la calma y el descanso, que eran asociados a la contemplación y opuestos a la actividad. "La raíz de skhole significaba, etimológicamente, parar o cesar; de aquí el tener reposo o paz. Más tarde significó tener tiempo desocupado o, específicamente, tiempo para uno mismo" (De Grazia, 1966, p. 2). La liberación de la ocupación muestra el ocio como una experiencia de tiempo con sentido a través de su liberación, más allá de la ocupación y más allá de un mero no estar ocupado, sino de dotar de sentido a esa liberación.

El ocio, sin importar la época, no deja de estar relacionado con el trabajo de forma complementaria. Siempre en el entendido de la ausencia de ocupación, ha de tener que ver con ella incluso hasta en su negación misma. En su raíz griega, skhole implica el estar libre de toda ocupación. Aristóteles presenta ya dicha relación en la Ética, bajo el ideal de que "trabajamos para tener ocio" (Aristóteles, 1985, p. 396, 1177b). Sobre esta frase, Josep Pieper, en El ocio y la vida intelectual, afirma que: "La frase traducida literalmente es la siguiente: estamos no ociosos para tener ocio" (Pieper, 1979, p. 12). La frase hace énfasis en el carácter liberador del ocio y parece mostrar el ocio como un tiempo con sentido a través de su liberación, más allá de la ocupación y más allá de un mero no estar ocupado sino de dotar de sentido a esa liberación. Eso ha tenido relevancia en la concepción actual, que acontece como el resultado de dos condiciones que se interrelacionan y preceden a nuestro siglo. Por un lado, está el avance industrial de la técnica, enfocado hacia la producción, el cual vino a traer más tiempo libre de ocupaciones. Por otro, encontramos 
que "el ocio forma parte integrante de la civilización técnica [...] el ocio es en sí una creación de la civilización industrial" (Dumazedier, 1964, p. 49). Ello responde a que la división del trabajo como consecuencia del capitalismo industrial, desde sus inicios hasta nuestros días, no ha cesado en su conquista por el tiempo. Se observa ya en la definición de valor que Marx estipulaba en las mercancías, el cual era basado en el tiempo de trabajo.

Ante tal conquista promovida por lo que habría sido un afán por la producción, que más tarde lo sería por el consumo, tuvo lugar la reivindicación hecha por los trabajadores en el siglo xix. A través de movimientos sociales, se exigió, como parte de sus derechos, tiempo de no trabajo mediante la reducción de la jornada laboral. Esa primera reivindicación fue alentada por el yerno de Marx, el filósofo cubano-francés Paul Lafargue (2010). En su obra El derecho a la pereza arremete contra las condiciones de trabajo de aquel entonces, en vista de una reivindicación del tiempo de trabajo orientada a su reducción con la intención de valorar y defender la importancia del ocio ${ }^{1}$. Algunos años más tarde, Bertrand Russel retomaría tal mensaje para darle continuidad y plasmarlo en su Elogio a la ociosidad. Ambos contribuyeron a la constitución de las condiciones materiales de la época presente. De modo que

[...] este logro permitió cuestionar el sentido del propio tiempo libre y volver la mirada al ocio clásico, un ocio que ya conocía el potencial de sentido y realización personal que aportan las experiencias desinteresadas y gustosas. El ascenso vertiginoso del tiempo libre vino a confundir tiempo y ocio, hasta tal punto que este cruce conceptual constituye uno de los aspectos característicos de esa época. (Cuenca y Aguilar, 2009, p. 14)

Pese a las reivindicaciones por el uso del tiempo propio, este influye en que el ocio sea comúnmente confundido con el tiempo libre. Frente a la confusión entre ambos términos, conviene aquí recordar la distinción que ofrece Sebastián De Grazia en Tiempo, trabajo y ocio:

Todo el mundo puede tener tiempo libre, y no todos pueden tener ocio. El tiempo libre es una idea de la democracia realizable; el ocio no es totalmente realizable, y, por tanto, es un ideal y no sólo una idea. El tiempo

1 “[...] el trabajo solo se convertirá en un condimento de placer de la pereza, un ejercicio benéfico para el organismo humano, una pasión útil para el organismo social en el momento en que sea sabiamente reglamentado y limitado a un máximo de tres horas por día [...]” (Lafargue, 2010, p. 29). 
libre refiere a una forma determinada de calcular una determinada clase de tiempo; el ocio es una forma de ser, una condición del hombre, que pocos desean y menos alcanzan. (De Grazia, 1966, p. XIX)

Por esto último, respecto al ocio como forma de ser, Aristóteles se refería a él como capacidad, en tanto que es necesario poder estar en la capacidad de tener un ocio decoroso ${ }^{2}$. No obstante, ocio y tiempo libre tienen en común el ejercicio de la libertad; así como el juego, que en ellos surge, son llevados a cabo por la voluntad. Esta capacidad está ligada a la formación del ocio. Aun actualmente, es posible plantearse la pregunta que planteaba Aristóteles: ¿en qué debemos emplear nuestro ocio?

La naturaleza misma procura no solo el trabajo adecuado sino también estar en capacidad de tener un ocio decoroso, el cual es, para decirlo de nuevo, el principio de todas las cosas. Siendo ambos necesarios, el ocio es, con todo, preferible al trabajo y tiene razón de fin, por lo cual hemos de investigar cómo hemos de emplear nuestro ocio. Seguramente que no en jugar, porque entonces el juego sería necesariamente el fin de la vida, lo cual es imposible. Los juegos, en efecto, deben practicarse más bien en conexión con los trabajos (porque el trabajador ha de dar un descanso a su fatiga y el juego es para descansar, mientras que el trabajo va acompañado de fatiga y esfuerzo). Por esto hay que introducir los juegos, pero vigilando la oportunidad de su empleo, como si aplicáramos una medicina, porque la actividad del juego es un relajamiento del alma, y de este placer resulta el descanso. Pero el ocio parece encerrar en sí mismo el placer, la felicidad y la vida bienaventurada”. (Aristóteles, 2000, p. 302. 1337b30 a 35-1338a5)

A este respecto, es evidente la distinción que hace Aristóteles entre el juego y el ocio: sitúa al ocio en una esfera superior y ubica al juego en asociación al descanso, ya sea físico o mental. El juego tiende a ser un descanso para la mente por traer consigo diversión y entretenimiento. Por ejemplo, el juego, en el caso de los deportes, puede implicar algún esfuerzo físico a la vez que una relajación para la mente. Sin embargo, pese a ser necesarios y estar unidos al trabajo, tienen como fin el relajamiento y la reposición de fuerzas tanto físicas

2 "La naturaleza misma procura no solo el trabajo adecuado sino también estar en capacidad de tener un ocio decoroso, el cual es, para decirlo de nuevo, el principio de todas las cosas" (Aristóteles, 2000, p. 302). 
como mentales. Por ello, son parte de un medio, al igual que el trabajo y a diferencia del ocio, que es un fin en sí mismo.

Para los antiguos griegos, el ocio llegó a ser una actividad propia de los nobles y solo para los hombres libres. Para Aristóteles, la distinción entre actividades nobles y serviles no era una cuestión de derecho para todos los ciudadanos; el poder llevar una vida de ocio era solo para hombres libres y no para esclavos, y se refirió a hombres también en cuanto a género, pues las mujeres no eran consideradas como seres libres. El optar por dar mayor valor a una actividad que no produce bienes útiles nada tenía entonces que ver con un sistema económico esclavista. La supremacía de la contemplación sobre la actividad no era una cuestión entonces de valor hacia los objetos o lo útil, sino de modos de vida, de cuál era el mejor de los modos de vida, o bien, el más valioso, a partir del tipo de actividad por el que habría de decantarse. De esta manera, el ocio como fin en sí mismo era también valorado así bajo el entendido de que "quien realiza una actividad cuyo fin es su propio ejercicio, se perfecciona a sí mismo. Esta es en última instancia la justificación de la distinción valorativa entre actividades nobles y serviles" (Cuenca 2013, p. 451). El dar más valor a un cierto tipo de actividades que a otras contenía también una inclinación a valorar más las actividades que apostaban por cultivar los modos de ser.

Siglos más tarde fue democratizado y establecido como un derecho ${ }^{3}$, de manera que pasó a ser una actividad para los trabajadores, puesto que la necesidad de trabajar se extendió a toda la esfera de la sociedad. Así también la del ocio y el tiempo libre: "El papel que la modernidad reserva al ocio no puede ser sino residual [...] solo se considera lícito el ocio en cuanto descanso conducente a la recuperación de la fuerza laboral" (Cuenca, 2013, p. 457).

Actualmente, el ocio es concebido como "tiempo para la realización humana" ${ }^{4}$, pese a continuar en una debacle entre un tipo de ocio para la realización y el ocio alienante del consumo, que es por lo común el que se lleva

3 Siguiendo este orden de ideas, puede observarse que el tiempo de trabajo funciona en relación con el tiempo libre, el cual es considerado un derecho humano. Según el artículo 24 de la declaración universal de los derechos humanos: "Toda persona tiene derecho al descanso, al disfrute del tiempo libre, a una limitación razonable de la duración del trabajo y a vacaciones periódicas pagadas” (Declaración Universal de los derechos humanos, 1948.

4 Joffre Dumazedier define el ocio como "el contenido del tiempo orientado hacia la realización de la persona como fin último". Ce contenu du temps orienté vers la réalisation de la personne comme fin dernière (1947, p. 93). 
a cabo en función del trabajo, como mero goce y divertimento para mitigar el desgaste producido por el trabajo.

\section{Del ocio al juego: una lectura del sentido de liberación}

Ante lo ya expuesto en cuanto a la noción y distinción de lo que es el tiempo libre, es menester plantear otros conceptos que se derivan del uso del tiempo libre y se desarrollan en la experiencia de ocio, a saber: juego, descanso, y recreación. Se afirma que estos son derivados del tiempo libre por el hecho de tratarse de actividades que se realizan en el periodo del tiempo libre, es decir, tras encontrarse desprovisto de la obligación del tiempo de trabajo. En este sentido, las tres actividades en mención implican la reposición de las fuerzas de trabajo, tanto en lo físico como en lo mental y lo espiritual. Por tanto, ellas han de contribuir a ser medios para llegar a un fin. Pero lo que nos ha de importar aquí es el sentido de ese fin, que ha de ser pauta del cómo se emplean el juego y la recreación, es decir, hacia qué fin han de orientarse.

Ocio y trabajo han existido de forma complementaria pese a las tergiversaciones más recientes en la relación de ambos términos. La relación del juego con estos corre, por tanto, por los mismos cauces. A lo largo de la historia de la humanidad se ha visto la necesidad imperiosa por el trabajo en concreto, por la actividad. Los filósofos griegos debatieron por años sobre cuál era el mejor de los modos de vida, la vida activa o la vida contemplativa. Si la actividad es inherente al género humano, lo es aún más el detenimiento, la contemplación, una necesidad por la pausa, el descanso y la demora. Es evidente, entonces, que el trabajo, la acción, lo laborioso, son inherentes a la vida misma. Ya el mismo cuerpo, su crecimiento y desarrollo de habilidades físicas y cognitivas es una labor de nuestra propia naturaleza. Cabe destacar la postura de Arendt:

Labor es la actividad correspondiente al proceso biológico del cuerpo humano, cuyo espontáneo crecimiento, metabolismo y decadencia final están ligados a las necesidades vitales producidas y alimentadas por la labor en el proceso de la vida. La condición humana de la labor es la vida misma. (Arendt, 2015, p. 21)

Es entonces posible afirmar que la labor corresponde a un movimiento natural, mientras que el trabajo sigue las exigencias de las necesidades creadas por el hombre en producir y consumir. Hanna Arendt, en su obra La condición 
bumana, se refiere con la expresión "vita activa" para designar tres actividades fundamentales: "labor, trabajo y acción” (Arendt, 2015, p. 21). Esas tres actividades resultan imprescindibles y totalmente necesarias para la vida, pero sin olvidar nunca sus igualmente necesarios complementos: juego, descanso y ocio.

El juego y el descanso son efectuados en el ocio y en el tiempo libre. Asimismo, se dimensionan de manera muy similar en virtud de la evolución de la ciencia y el uso de la técnica, según ha sido en cada época, pues "históricamente el ocio es la skholé griega, aunque se puede decir que ha existido siempre y de diversa forma, mientras que la concepción de tiempo libre arranca en el desarrollo industrial" (Cuenca, 2000, p. 20). El ocio sigue siendo en gran medida confundido, relacionado y hasta entendido como tiempo libre.

En la actualidad, los avances tecnológicos nos han dado la posibilidad de tener más tiempo libre, el cual puede ser destinado a la recreación en función de una mayor diversidad en las opciones del juego: la capacidad de poseer mayor tiempo para sí mismo concede mayor posibilidad de desarrollar las capacidades creativas y de relación con el juego (Cuenca, 2009). En concreto, hemos de entender el juego aquí "como una actividad recreativa que proporciona descanso, distracción, diversión y recuperación. Como alivio del trabajo" (McPhail, 1999, p. 86). La posibilidad de poder tener más tiempo libre da mayores condiciones para el ocio y, por ende, también para el juego. Por eso existe una tendencia a concebir la sociedad actual como una sociedad ociosa.

El juego se da en el ocio en conjunto con la recreación, y llega a compartir el sentido de esta. Es decir, el juego también puede tener sentido de recreación. Para ahondar más en ello, es preciso definir qué es la recreación, por lo que haremos referencia aquí a dos conceptos, de los cuales el primero vincula la recreación como ocio. En primera instancia, nos adherimos a la comprensión del concepto en cuestión a la perspectiva de la recreación de Manuel Cuenca (2009) que versa en un sentido más profundo que el de la mera diversión, la alegría o el goce. De ahí también su conexión con el ocio, que, desde la óptica de Kriekemans (1973), también se entiende como una recreación que tiene como fin la recuperación del ejercicio de la voluntad y el valor de vivir. Ante la postura expuesta, es también necesario ofrecer un concepto general de la recreación, esto con la finalidad de enfatizar los aspectos de ella que sean renuentes al análisis que aquí se pretende llevar a cabo: 
La recreación es un conjunto de ocupaciones a las que el individuo puede entregarse con pleno consentimiento, ya sea para descansar, divertirse, desarrollar su participación social voluntaria, su información o formación desinteresada, después de haberse liberado de toda obligación profesional, familiar, social”. (Minello, 1983, p. 12, citado por McPhail, 1999, p. 86)

La importancia actual del juego en la recreación, como actividad de integración del tiempo libre, considerado derecho, es un signo que decreta la necesidad del reposo productivo y el aumento de su calidad en vista del valor del descanso. No obstante, el goce que implica el juego provee de elementos para la realización que lo distinguen como actividad recreativa en una orientación positiva dentro del ocio. De este modo, el juego, pese a ser un medio para el descanso y la recreación, del cual puede disponerse a través del ocio, es también un medio que, orientado hacía el cuidado de sí, puede prescindir de criterios utilitarios en función de generar valor de cambio. Es decir, puede darse como actividad no utilitaria, a pesar del continuo riesgo de ser mercantilizado hacía el lucro y el vicio.

Encausar toda concepción de ocio hacia el consumo viene de la mano con una tendencia reduccionista de limitar la noción de ocio como mero entretenimiento y diversión. Ya que el ocio es condición de posibilidad para el juego, existe cierta tendencia a verlos de manera inseparable, lo cual se deriva del afán por la diversión que es proveedora de evasión, que a su vez suelen obtenerse en el descanso y la recreación. Con esto no queremos decir que el ocio no deba proveernos de diversión por medio del juego, sino que lo que ha de cuidarse es el sentido de este, su orientación, de manera que ella se enfoque a la realización y libre juego de las potencialidades y capacidades del individuo, y no a un mero reducto reparador de fuerzas abnegado a la necesidad y obligación en función del trabajo. Vemos entonces con claridad la desvalorización del ocio cuando se le ubica como contenedor de diversiones alienantes que implican una consecuencia más de la relación entre ocio y consumo ${ }^{5}$.

El concepto de ocio, situado en la actualidad en relación con su común asociación con el juego y, por ende, con el entretenimiento, supone una integración en el sentido de la definición de ocio de Dumazedier: "Ce contenu du temps orienté vers la réalisation de la personne comme fin dernière"

5 "El problema surge cuando la diversión, como única referencia de ocio, se transforma en medio de manipulación y enajenación” (Cuenca, 2014, p. 63). 
$(1947 \text {, p. 93 })^{6}$. La inserción del ocio a la industria del consumo representa una amenaza en su orientación si su sentido ha de ser la realización de la persona. Si el ocio no es bien orientado, las actividades que surjan en él, como el juego y la recreación, también se verán afectadas por una ausencia en la orientación del ocio hacia la realización de la persona. De ahí surge la necesidad de que los momentos del tiempo libre, del consumo, las experiencias de ocio y el juego se ocupen en acciones dirigidas a objetivos edificantes. Mediante el juego puede ejercerse el cuidado de la salud a través de la actividad física; mediante la lectura, puede cultivarse la reflexión. Por ejemplo, en el goce al contemplar una pieza artística, o bien al llevar a cabo su creación, se ponen en libre juego las facultades y potencialidades humanas, lo cual puede considerarse un consumo del tiempo libre, en tanto que experiencia de ocio dirigida hacia la revalorización de lo humano.

Como se ha visto, el ocio ha estado siempre en una constante relación con el juego y el arte. Sin embargo, arte y juego, pese a que suelen ser confundidos, no conllevan los mismos fines. El ser humano es capaz de educar su capacidad de tener ocio, de formarse en las artes, educando su sensibilidad y ampliando su dimensión estética y sensorial que mantiene relación con su interés por el juego. Schiller (1990) dio cuenta de ello en sus Cartas a la educación estética del hombre. En ellas pretendió conciliar la unión de la sensualidad y la razón en el arte y el juego como elementos de exaltación de la naturaleza humana: "El hombre no debe hacer con la belleza sino jugar, y debe jugar solo con la belleza [...] el hombre solamente juega cuando, en el sentido completo de la palabra, es hombre y solamente es hombre completo cuando juega" (Schiller, 1990, pp. 92-93) ${ }^{7}$. Con ello, Schiller pretende reivindicar el juego, la belleza ${ }^{8}$ y los sentidos en una exhortación de lo grandes y valiosas que pueden ser

6 "El contenido del tiempo orientado hacia la realización de la persona como fin último". Traducción del autor.

7 Cuenca (2014) nos comenta sobre el aporte de Schiller respecto a la reflexión en torno a la experiencia de ocio, que pretende resaltar la seriedad del juego estético a fin de concebirlo como algo más allá de una mera diversión banal. Con esto se destaca el valor mediador de la vivencia estética en el juego como agente que contribuye en la realización y desarrollo integral del ser humano.

8 De la belleza dimana un sentido no utilitario, que permite en su apreciación la posibilidad del desarrollo de las capacidades humanas (Cuenca, 2014). De aquí la necesidad de que el arte como expresión de la belleza, y el juego como condición de posibilidad de la belleza tengan en común aporte el tener por esencia un sentido no utilitario y, en cambio, una exaltación por un 
las capacidades humanas cuando son desarrolladas a través de la educación estética, como una exaltación de la dignidad y libertad del hombre que puede darse también a través del ocio.

El juego ha sido una experiencia dada en el paso de la civilización. Cada cultura ha tenido sus respectivos juegos. Estos siempre han perseguido la búsqueda de un aporte compensatorio a la vez que el goce de la experiencia que aporta el hecho de efectuar el juego'. Los juegos, como en el caso de los deportes, pueden llegar a similitudes con el arte, pues se trata en ambos casos de experiencias sensoriales. Sin embargo, el juego requiere de reglas, mientras que, en el arte, sobre todo en el arte contemporáneo, se ha estado difuminando con respecto al detrimento en la técnica y ensalzamiento de la representación y el discurso. En lo que a ello respecta, no entraremos aquí en debate en cuanto a definiciones y cánones. Así, aunque arte y juego pueden ser un deleite, no lo son de la misma manera. El arte y la experiencia estética, a pesar de no estar bajo el influjo de ciertas reglas o técnicas, no tienen como fin el mero divertimento; en cambio, el juego sí implica necesariamente la diversión. Por ello, sumando a la distinción entre los conceptos, el arte es análogo al juego (Cuenca, 2014, p. 53).

Sin embargo, lo que nos interesa es resaltar esa similitud entre arte y juego, de una experiencia autotélica, lo cual quiere decir que se lleva a cabo sin ninguna pretensión de utilidad ni ganancia, o más allá de lo gratificante de la experiencia misma. Aquí el juego y el arte trastocan una característica del ocio que nos ha interesado recuperar, el valor y elogio de lo improductivo como un rechazo de la utilidad. El no buscar la utilidad en la experiencia es condición esencial del arte, que por sí mismo no tiene utilidad ni tiene por qué tenerla. En el caso del ocio, este es un fin en sí mismo y no persigue la utilidad; su ganancia es percibida en bienes inmateriales que pueden ser relativos a la

tipo de expresión que contribuye a enriquecer el valor de lo que es el ser humano.

9 Teniendo en cuenta que actualmente se sabe que la mayoría de los mamíferos juegan y que no es en sí una actividad propia del ser humano, aquí nos referiremos solo a la relación de la experiencia del juego en el ocio y su relación con la experiencia estética. Huizinga señala al respecto que: "El juego es más viejo que la cultura; pues, por mucho que estrechemos el concepto de ésta, presupone siempre una sociedad humana y los animales no han esperado a que el hombre les enseñara a jugar. Con toda seguridad podemos decir que la civilización humana no ha añadido ninguna característica esencial al concepto de juego. Los animales juegan, lo mismo que los hombres. Todos los rasgos fundamentales del juego se hayan presentes en el de los animales" (Huizinga, 2007, p. 11). 
contemplación y el ejercicio del pensamiento, así como a la introspección, la espiritualidad, el cultivo de la mente a través del estudio o bien de la ejercitación física o ejecución de una expresión artística. En estos últimos aspectos se liga al juego, que suele ser más un medio de descanso ante el trabajo, tanto para el cuerpo como para la mente.

"El ocio, cualquiera que sea su función, es, en primer término, una liberación y un placer. Luego se separa en tres categorías que, en nuestra opinión corresponden a sus tres funciones primordiales de descanso, diversión y desarrollo" (Dumazedier, 1964, pp. 30-29). Sin embargo, en la actualidad dichas funciones del ocio que pueden converger con el juego han sido embestidas por la mercantilización. La sociedad de consumo vicia el aporte de los juegos hacia la mera producción de valor de cambio. Esto ha afectado mucho al ocio en tanto que lo ha degradado a ser visto como un tiempo residual de mera diversión con una connotación que puede ser peyorativa y nociva.

Los juegos son fuente directa de diversión y la industria se ha aprovechado por medio del negocio de las apuestas, que, si bien han tenido presencia en épocas anteriores, nunca había sido de manera tan global. Los deportes han desvirtuados de su carácter estético y lúdico, más aún por ser despojados de su verdadero sentido de convivencialidad y socialización. Han sido orientados más al negocio que a la libertad y el descanso en vistas de la realización. El juego por medio del deporte ofrece aportaciones éticas para las personas. Implica la promoción de ciertos valores inculcados por las mismas reglas del juego: el juego limpio, la jovialidad de la competencia, y el respeto como promotores de dignidad humana en la actividad del juego. En el caso de los videojuegos, se han arraigado a la industria del ocio a través del consumo de manera nociva sin una prescripción o mesura de sus límites en su uso. Es indudable que generan comunidades enteras interconectadas a una interfaz que las une desde cualquier parte del mundo. Asimismo, estos juegos se muestran como una especie de narrativa en sus contenidos, por la complejidad que sus tramas pueden llegar a contener posibles enseñanzas éticas al mismo tiempo que logran ser fuente de experiencias estéticas y alusiones artísticas por sus avanzados gráficos. Sin embargo, existe en todo ello una evidente propensión a degenerarse en vicio.

El juego, al darse durante el ocio, implica libertad, cuya característica hemos señalado ya y que sirve de fundamento para afirmar que el ocio tiene una dimensión lúdica. Ella conjuga con una de las funciones del ocio, la del 
descanso ${ }^{10}$. En el juego se da la recreación y esta, a su vez, se encuentra inmersa en el ocio, en la medida que regenera y provee del descanso, el cual es uno de los beneficios del juego mismo. A este respecto, Cuenca (2009) señala que el juego es entendido como un agente importante dentro de la dimensión lúdica del ocio, y es indispensable para el obtener una estabilidad física y mental que favorezca el desarrollo de las facultades de cada individuo. Esa alternancia y ejercitación propias de la actividad del juego toman partida en la recreación donde el individuo hace uso de sus libres facultades a fin de recrearse, en función no solo de su descanso ante el esfuerzo del trabajo, sino también de su gratificación y satisfacción.

Ocio, juego y arte pueden coincidir en otorgar un sentido de liberación en relación con el trabajo. En este sentido, es posible afirmar que el ocio y el juego tienen un sentido liberador en función de su relación con el trabajo, como una relación análoga a la realidad. Desde esta idea podemos acudir a Marcuse (1983) el cual trae al diálogo alusiones a Schiller (1990) en que

[...] el hombre es libre solo cuando está libre del constreñimiento, externo e interno, físico y moral —cuando no está constreñido ni por la ley ni por la necesidad. Pero tal constreñimiento es la realidad. La libertad es, así, en un sentido estricto, liberación de la realidad establecida: el hombre es libre cuando la «realidad pierde su seriedad» y cuando su necesidad llega a ser ligera. (Marcuse, 1983, p. 175)

Tanto el ocio como el juego suponen libertad de la necesidad del trabajo, una experiencia que aparta de la tensión de la necesidad. Así, el individuo puede desarrollar libremente la potencialidad de sus capacidades. El juego no solo puede entenderse en términos de vacilación o vagancia, lo cual sugiere un aspecto residual. Nos referimos más bien a la libertad del juego en tanto que, además de voluntario, aporta una gratificación: “Jugar aporta algo al jugador; por eso juega. Pero la recompensa fundamental reside en la experiencia de la propia actividad (sea cual sea)" (Black, 2013, p. 15). Dicha libertad y dichas capacidades pueden desplegarse a su vez en conjunto con el juego, que, como reposición del desgaste y alienación del trabajo, llevan al sujeto a la búsqueda

10 Una de las funciones menos discutidas del ocio es su función regeneradora, consecuencia del descanso (Cuenca, 2009, p. 100). 
de su recreación en función del goce, la experiencia y la retribución de lo gratificante de su tiempo de no trabajo:

La realidad que "pierde su seriedad» es la inhumana realidad de la necesidad y el deseo insatisfecho, y pierde su seriedad cuando la necesidad y el deseo pueden ser satisfechos sin trabajo enajenado. Entonces, el hombre es libre para "jugar" con sus facultades y potencialidades y con las de la naturaleza, y sólo "jugando" con ellas es libre. Su mundo entonces es el despliegue y su orden el de la belleza. Porque es la realización de la libertad, el juego es más que la constreñida realidad física y moral. (Marcuse, 1983, p. 175)

El juego dentro del ocio implica, por tanto, la liberación. Si el trabajo impera en forma de actividad incesante por necesidad, el ocio se arraiga en el ámbito de la libertad. En la libertad acontece el juego e invita al desentendimiento de la necesidad para la recreación del individuo, con lo cual puede aportarse a sí mismo, por medio de sus sentidos, su propia potencialidad:

El campo de la libertad se ve yaciendo más allá del de la necesidad: la libertad no está dentro sino fuera de la "lucha por la existencia". La posesión y el abastecimiento de las necesidades de la vida son el prerrequisito, antes que el contenido, de una sociedad libre. El campo de la necesidad, del trabajo, es un campo de ausencia de libertad porque en él la existencia humana está determinada por objetivos y funciones que no le son propios y no permiten el libre juego de las facultades y los deseos humanos. (Marcuse, 1983, p. 180)

En este sentido, el juego trae libertad ${ }^{11}$. El sentido liberador del juego desplaza la necesidad para enfocarse en el desenvolvimiento de las capacidades, en la realización y la recreación de sí. Aquí Marcuse (1983) atiende a la reflexión por el juego desde una posición en favor de la percepción de la realidad a partir de la dimensión estética. Con ello, muestra su fuerte influencia en la obra de Schiller (1990), quien abogaba por una educación estética del hombre

11 El juego es una actividad libre por definición. La libertad es parte esencial de su razón por la cual ha de ser llevado a cabo. Y es en esa libertad donde es posible experimentar determinada satisfacción. La libertad y la satisfacción constituyen los motivos que dotan de sentido a la actividad lúdica. Por lo tanto, el juego es efectuado debido a la satisfacción que resulta de su ejercicio mismo (Cuenca, 2009). 
basada en la confluencia entre la razón y la sensibilidad. Aquí el sentido liberador del juego se hace presente en vencer el imperativo de la necesidad y la fatiga del trabajo. Es posible detectar este sentido liberador del juego en que: "La transformación de la fatiga (el trabajo) en juego, y de la productividad represiva en 'despliegue' —una transformación que debe ser precedida por la conquista de la necesidad (la escasez) como el factor determinante de la civilización" (Marcuse, 1983, p. 179).

\section{Reflexiones finales}

Jugar siempre ha sido un escape de la realidad más que necesario: es irremediable e inevitable ${ }^{12}$. Seguimos jugando. Pese a que tanto humanos como animales juegan, solo a los seres humanos les surge la necesidad de un tiempo para sí mismos, la necesidad del ocio. Esta emerge de "la tensión, que se acrecienta con la racionalización y tecnificación del trabajo, además de acrecentar la fatiga, plantea paralelamente una mayor necesidad de diversión y tiempo privado, un tiempo para sí” (Cuenca, 2009, p. 286). Durante el trabajo se nos expropia nuestro tiempo. Lo vendemos para obtener una ganancia con la cual subsistir. El tiempo que nos resta ha de ser incluso más valioso por ser un tiempo destinado no al imperativo de la obligación y la necesidad de la subsistencia y manutención, sino de nuestra realización.

Más allá de percibirlo como un tiempo libre, que es libre solo en función del trabajo, hemos de considerarlo como un tiempo propio usado a voluntad para la libertad, la preservación de nuestra dignidad, el descanso a través del juego y la recreación, la diversión, y el desarrollo de nuestras capacidades. Un tiempo para el ocio. Es decir, el ocio entendido como una experiencia dentro de un uso del tiempo a voluntad, un ejercicio de autonomía y libertad que se conjuga con el juego como un tiempo en el cual el individuo se recrea a sí mismo, más allá de una mera reposición de fuerza de trabajo (Cuenca 2009). El juego y el ocio han de seguir manteniéndose en el carácter de lo necesario,

12 La actividad del juego es voluntaria y promotora del ejercicio de la libertad; ofrece diversión y aligeramiento de la realidad mientras se lleva a cabo, de ahí que se llegue a afirmar que el juego distrae, y en tanto lo hace libera. Por ello nos provee de descanso y nos ofrece una distancia ante la realidad que nos libera de ella y permite impulsar nuestra capacidad creadora (Cuenca. 2009). 
como actividades vitales de relevancia ante el imperativo por la obligación y la necesidad del trabajo. El juego se da de manera voluntaria dentro de la experiencia de ocio, la cual nunca es impuesta, bajo el entendido de que el ocio y el juego han de complementarse de manera armoniosa y equilibrada. Es decir, no como un mero medio para descansar del trabajo sino para el beneficio y realización de los seres humanos en un sentido liberador, el cual es posible en el ocio a través del juego y la recreación.

\section{Referencias}

Arendt, H. (2015). La condición humana. Madrid: Paidós.

Aristóteles. (1985). La ética nicomaquea. Barcelona: Gredos. (Original publicado en el s. IV a. e. c.)

Aristóteles. (2000). La política. México: Editorial Porrúa. (Original publicado en el s. IV a. e. c.)

Black, B. (2013). La abolición del trabajo. Logroño: Pepitas de calabaza.

Cuenca, M. (2009). Ocio humanista. Bilbao: Universidad de Deusto.

Cuenca, M. y Aguilar, E. (eds.). (2009). El tiempo de ocio: transformaciones y riesgos en la sociedad apresurada. Bilbao: Universidad de Deusto.

Cuenca M. y Aguilar E. (2014). Ocio valioso. Bilbao: Universidad de Deusto.

Cuenca, J. (2013). La existencia valiosa. En A. Ortiz-Osés, B. Solares y L. Garagalza (eds.), Claves de la existencia. El sentido plural de la vida humana (pp. 449-470). Barcelona: Anthropos Editorial del Hombre. DoI: https://doi. org/10.24850/j-tyca-2018-01-01

De Grazia, S. (1966). Tiempo, trabajo y ocio. Madrid: Tecnos.

Dumazedier, J. (1947). Sociologie empirique du loisir critique et contre-critique de la civilisation du loisir, Paris: Editions du le Seuil. DoI: https://doi. org/10.2307/3320680

Dumazedier, J. (1964). Hacia una civilización del ocio. Barcelona: Estela.

Huizinga, J. (2007). Homo ludens. Madrid: Alianza Editorial.

Lafargue, P. (2010). El derecho a la pereza. Madrid: Público.

Marcuse, H. (1983) Eros y la civilización. Madrid: Sarpe.

Mc Phail, E. (1999). El tiempo libre y la autonomía. Una propuesta. La ventana, (9), 86: https://doi.org/10.17151/hpsal.2015.20.2.8 
Minello, N. (1983). Impacto de una política de bienestar, cultura y recreación para los trabajadores promovida por el Estado. En Cuadernos Laborales. México: Secretaría de Trabajo y Prevención Social.

Organización de las Naciones Unidas. (1948). Declaración Universal de los Derechos Humanos. DOI: https://doi.org/10.2307/j.ctv86dh99.6

Pieper, J. (1979). El ocio y la vida intelectual. Madrid: Rialp.

Schiller, F. (1990). Kallias: Cartas a la educación estética del hombre. Barcelona: Anthropos. 